\title{
Prédicteurs de détresse liée aux symptômes chez les femmes atteintes de cancer du sein durant le premier cycle de chimiothérapie
}

\author{
Par Marcia M. Boehmke et Jean K. Brown
}

\section{Abrégé}

Objectifs : Déterminer dans quelle mesure les caractéristiques personnelles et les "facteurs de personnalité " permettent de prévoir la détresse liée aux symptômes chez les femmes atteintes d'un cancer du sein, et ce, durant le premier cycle de chimiothérapie.

Devis : prospectif, longitudinal, corrélationnel.

Échantillon et milieu : 120 femmes atteintes d'un cancer du sein de stade I et II, sur le point de débuter leur premier cycle de chimiothérapie, ont été recrutées dans six établissements d'oncologie différents.

Méthodes : Des questionnaires d'auto-évaluation ont été remplis avant le début, au nadir et à la fin du premier cycle de chimiothérapie.

Principales variables de recherche : caractéristiques personnelles, facteurs de personnalité et détresse liée aux symptômes.

Observations : L'optimisme et le locus de contrôle externe prédisent des degrés faibles de détresse liée aux symptômes et ce, au nadir et à la fin du premier cycle. La fatigue, la modification de l'aspect physique et l'insomnie causent la plus grande détresse, les résultats de détresse liée aux symptômes les plus élevés ayant été signalés au nadir, avec un résultat moyen de 1,98 sur l'échelle de Likert en 5 points.

Conclusions : Les femmes qui conservaient un bon moral et faisaient confiance à leurs prestataires de soins éprouvaient des degrés moindres de détresse liée aux symptômes. Les résultats révèlent que la plupart des femmes ressentaient une certaine détresse liée aux symptômes, particulièrement au milieu du premier cycle de chimiothérapie.

Le diagnostic d'un cancer du sein provoque chez une femme un stress considérable, du diagnostic de la maladie jusqu'à la fin du traitement. Le cheminement de chaque femme atteinte d'un cancer du sein - et son traitement - est unique en son genre et il est désormais connu sous le nom de « détresse liée aux symptômes » (Rhodes et Daniels, 1999). Malgré les similitudes des symptômes et des effets indésirables des traitements, les réponses individuelles à ces symptômes peuvent varier grandement. Le dépistage des femmes les plus à risque d'éprouver de la détresse liée aux symptômes est primordial, car cette dernière peut, à des degrés élevés, nuire à la qualité de vie de celles qui ont été traitées pour un cancer du sein. Réduire au maximum la détresse liée aux symptômes durant le traitement pourrait permettre d'améliorer considérablement la qualité de vie. Le but de cette étude était de déterminer dans quelle mesure les caractéristiques personnelles et les « facteurs de personnalité » permettent de prédire la détresse liée aux symptômes chez les femmes atteintes d'un cancer du sein et ce, durant le premier cycle de chimiothérapie.

\section{Cadre théorique}

Cette étude est basée sur le modèle théorique du stress et de l'adaptation de Lazarus et Folkman, englobant les concepts du stress, de l'évaluation de la situation, de la réaction et des conséquences (Lazarus et Folkman, 1984). Ce cadre théorique intègre le concept d'évaluation cognitive entre l'apparition du stresseur (cancer du sein) et la résultante (détresse liée aux symptômes). Par l'évaluation cognitive, l'individu mesure la portée d'un événement éprouvant et décide si cet événement va toucher ou non son bien-être. Lazarus et Folkman ont démontré que les processus d'évaluation étaient influencés par les caractéristiques personnelles (telles que l'âge, la situation matrimoniale et le statut socio-économique) et les facteurs de personnalité (tels que l'optimisme et le locus de contrôle) et que ces derniers pouvaient modeler la réaction des individus face à l'événement. La relation entre les caractéristiques personnelles et les facteurs de personnalité en tant que prédicteurs de l'appréciation du diagnostic d'un cancer du sein par une femme, et la détresse liée aux symptômes qui en résulte, constituait l'objectif premier de cette étude. Cette théorie laissait entrevoir que l'évaluation d'une menace (cancer du sein) et la résultante (détresse liée aux symptômes) seraient liées à une combinaison de caractéristiques personnelles (âge, situation matrimoniale et statut socio-économique) et de facteurs de personnalité (optimisme et locus de contrôle).

\section{Revue de la littérature}

La détresse liée aux symptômes a été définie comme « le degré de malaise signalé par les patients par rapport à leur perception des symptômes ressentis » (McCorkle et Young, 1978) et comme « la détresse, ou souffrance, physique et morale qui résulte de l'expérience de la survenue du symptôme ou de la perception des états affectifs "(Rhodes et Watson, 1987). Les résultats des recherches ont démontré que la détresse liée aux symptômes pouvait restreindre l'autosoin, menacer l'indépendance, altérer les relations sociales et diminuer l'observation des protocoles de traitement et de ce fait, la survie (Degner et Sloan, 1995; Kukull, McCorkle et Driever, 1986).

Les recherches ont permis de dégager quatre variables en rapport étroit avec la détresse liée aux symptômes qui touche les patients atteints de cancer. Ces variables sont : l'âge, le sexe, le type de cancer et le traitement anticancéreux. Les patients plus jeunes (Cimprich, 1999; Degner et Sloan, 1995; Pasacreta, 1997; Sarna et Brecht, 1997) et les femmes (Degner et Sloan, 1995; Tishelman, Taube et Sachs, 1991) rapportaient des degrés plus importants de détresse liée aux symptômes. Certains types de cancer, tout spécialement le cancer du poumon, étaient associés à des degrés supérieurs de détresse liée aux symptômes. (Degner et Sloan, 1995; Sarna et Brecht, 1997). Il existait une corrélation entre des seuils de détresse liée aux symptômes plus élevés et le stade du

Marcia M. Boehmke, inf., DNS, ANP(c) est professeure adjointe en sciences infirmières à l'University at Buffalo - The State University of New York, aux États-Unis. Son principal champ de recherche est le cancer du sein, la détresse liée aux symptômes et les groupes de symptômes.boehmke@buffalo.edu

Jean K. Brown, inf., PhD, FAAN, est Doyenne associée, Affaires étudiantes, à l'University at Buffalo, Buffalo, NY. 
cancer (Ehlke, 1988), la récidive (Munkres, Oberst et Hughes, 1992) et la diminution de la survie (Kukull, McCorkle et Driever, 1986).

Compte tenu des degrés élevés de détresse liée aux symptômes rapportés par les patientes célibataires, on a postulé que la famille, de même que le soutien social, exerçait une influence bénéfique (Tishelman et al., 1991). La situation matrimoniale dans ses rapports avec la détresse liée aux symptômes n'a pas fait l'objet de beaucoup d'études.

De plus, les rapports de l'éducation et du statut socio-économique avec la détresse liée aux symptômes n'ont pas fait l'objet d'une attention suffisante. Une éducation supérieure pourrait être une variable significative, étant donné que des gens mieux éduqués seraient tentés de poser plus de questions et/ou de chercher à s'informer davantage sur leur maladie. Cette approche, axée sur la recherche d'informations ou la résolution de problème, pourrait à son tour faire varier dans un sens ou dans l'autre la détresse liée aux symptômes. Sarna et Brecht (1997) ont examiné les niveaux d'éducation et découvert que la confiance en l'avenir des patients les plus éduqués affichait des niveaux plus faibles sur l'échelle de mesure de la détresse liée aux symptômes (Sarna et Brecht, 1997). De même, le statut socio-économique peut avoir une incidence sur l'accès aux soins. Avec l'escalade des coûts de santé, un retard dans la prise en charge peut avoir des conséquences sur l'issue du traitement et la détresse liée aux symptômes ressentie par les patients. À ce jour, aucune étude n'a examiné le statut socio-économique.

Ehlke (1988) a étudié la détresse liée aux symptômes des femmes atteintes de cancer du sein et a établi un parallèle entre le locus de contrôle interne et une détresse liée aux symptômes atténuée. Cela corrobore les travaux de Lazarus et Folkman (1984), selon lesquels les caractéristiques personnelles et les facteurs de personnalité reflètent l'essence d'une personne et influencent son évaluation de la situation et sa réaction au stress.

Les variables de l'âge, du sexe, du type de cancer et du type de traitement ont été examinées dans le détail, contrairement à la situation matrimoniale, au niveau d'éducation et au statut socioéconomique, qui n'apparaissent que dans quelques études et dont le rapport avec la détresse liée aux symptômes n'a pu être établi que d'une manière spéculative. De même, les variables psychosociales de l'optimisme et du locus de contrôle, affectant la détresse liée aux symptômes, ont été inégalement étudiées chez les patients du cancer. Cela méritait d'être souligné car d'autres disciplines, comme la psychologie sociale et comportementale, ont découvert que l'évaluation de la situation, la perception et les attitudes influençaient l'optimisme et le locus de contrôle et entretenaient un rapport certain avec les événements stressants (Lazarus et Folkman, 1984). Des recherches sur l'optimisme et le locus de contrôle présenteraient un grand intérêt car la « perception » est un concept clé de nombreuses définitions de la détresse liée aux symptômes, à savoir, la « gêne en relation avec la perception » (McCorkle et Young, 1978) et la «perception des états affectifs »(Rhodes et Watson, 1987).

\section{Objectifs}

Le but de cette étude était de déterminer dans quelle mesure les caractéristiques personnelles et les facteurs de personnalité pouvaient prédire la détresse liée aux symptômes chez les femmes atteintes d'un cancer du sein, durant le premier cycle de chimiothérapie. Deux questions de recherche étaient posées : a) Dans quelle mesure les caractéristiques personnelles (âge, situation matrimoniale, statut socio-économique) et les facteurs de personnalité (optimisme et locus de contrôle) permettent-ils de prédire la détresse liée aux symptômes au nadir et à la fin du premier cycle de chimiothérapie et modifient-ils les scores attachés à celle-ci durant la même période? b) Quelles sont la fréquence des symptômes et l'intensité de la détresse liée aux symptômes durant le premier cycle de chimiothérapie?

\section{Méthodes}

\section{Devis et contexte}

Un devis prospectif, longitudinal, corrélationnel a été utilisé pour recruter des femmes de six établissements : cinq cabinets privés d'oncologie médicale (quatre situés dans la banlieue de Buffalo, État de New York, et un situé dans l'agglomération de Buffalo) et le Erie County Medical Center, une clinique publique d'enseignement d'oncologie médicale située dans la ville de Buffalo. Ces six établissements ont été choisis pour que l'échantillon reflète une diversité éducative et socio-économique. Les femmes récemment diagnostiquées d'un cancer du sein et sur le point de débuter une chimiothérapie ont été suivies pendant le premier cycle de chimiothérapie adjuvante. Le cadre temporel délimitant cette étude a été décidé en fonction d'une correspondance personnelle avec Ruth McCorkle, RN, PhD (correspondance personnelle, 10 février 2001). Mme McCorkle a émis l'hypothèse selon laquelle les femmes éprouvaient le plus d'effets secondaires et conséquemment le plus de détresse liée aux symptômes, durant les 7 à 14 premiers jours (nadir) suivant le début de la chimiothérapie. Cependant, peu de recherches ont étudié le nadir du cycle de chimiothérapie.

\section{Échantillon}

L'échantillon de convenance était composé de 120 femmes. Les critères d'inclusion étaient : cancer du sein de stade I ou II avec confirmation histologique, être sur le point de commencer une chimiothérapie adjuvante, avoir plus de 18 ans, avoir une maîtrise de l'anglais lu et parlé, et être capable de comprendre et de remplir le consentement éclairé et les questionnaires. Les critères d'exclusion étaient : une malignité préexistante ou concomitante, la présence de tout autre type de traitement adjuvant, une affection comorbide incapacitante (sclérose en plaques, BPCO, arthrite) et considérée par la principale investigatrice (PI) comme pouvant influencer le degré de détresse liée aux symptômes des patientes en plus du cancer du sein, des antécédents ou une maladie psychiatriques (documentés dans le dossier médical ou révélés par un traitement médicamenteux), le fait d'avoir vécu un événement particulièrement dramatique dans les 30 derniers jours (divorce, décès dans la famille, perte d'emploi, etc.) ou incapacité de remplir correctement les questionnaires de l'étude. La taille de l'échantillon de 120 était basée sur une puissance de 0,80 , une valeur de l'effet moyenne et un alpha de 0,05 pour une régression multiple avec six variables indépendantes, comme le suggère Cohen (1992).

\section{Instruments}

Détresse liée aux symptômes. La détresse liée aux symptômes a été mesurée au moyen de la Symptom Distress Scale (SDS) de McCorkle, une échelle de Likert en 5 points comprenant 13 items, avec des valeurs allant de 13 (faible détresse) à 65 (forte détresse) (McCorkle et Young, 1978). La cohérence interne et la fiabilité de test-retest de la SDS sont bien établies. Dans cette étude, le coefficient alpha pour les 3 points de collecte des données était respectivement de 0,$82 ; 0,80$ et 0,80 . Cet instrument a été largement utilisé pour mesurer la détresse liée aux symptômes chez les malades du cancer et sa validité est notoirement établie.

Âge et situation matrimoniale. L'âge et la situation matrimoniale des participantes ont été obtenus à partir du dossier médical ou lors d'une entrevue.

Statut socio-économique. Le statut socio-économique a été calculé selon l'indice du statut social à deux facteurs d'Hollingshead (Hollingshead, 1975). Cette formule a été choisie parce qu'elle intégrait l'éducation et la profession et avait été élaborée à partir d'estimations des positions occupées par les individus au sein d'une structure sociale complexe, industrielle et urbaine. L'éducation est opérationnalisée en terme de nombre d'années de scolarité complétées et notée sur une échelle à 7 points allant de « en dessous 
de la 7e année » (score de 1) jusqu'à « diplôme supérieur d'études professionnelles ou universitaires » (score de 7). La profession est évaluée sur une échelle comportant 9 degrés, en fonction des titres et des codes de professions de l'US Census (1970). L'échelle de 9 échelons s'étend de «cadres supérieurs, patrons de grandes entreprises et professions libérales de premier ordre » (9) jusqu'à « ouvriers agricoles/employés subalternes » (1).

La cote du statut socio-économique a été calculée en multipliant l'éducation par un coefficient de pondération de 3 et la profession par un coefficient de 5; la somme des cotes fournit la cote correspondant au statut. Les cotes établies d'après cet indice peuvent varier de 8 à 66 , un score supérieur correspondant à un rang social plus élevé (Hollingshead, 1975). Malgré l'absence d'écrits sur la fiabilité et la validité de cet instrument, il a été largement utilisé comme mesure du statut socio-économique. Par souci d'exactitude, seule la PI était habilitée à attribuer les scores aux sujets de cette étude, et les calculs ont été vérifiés une nouvelle fois pour confirmer les scores obtenus au moment de l'entrée des données.

Optimisme. L'optimisme a été mesuré à l'aide du Life Orientation Test (LOT) de Scheier et Carver (Scheier et Carver, 1985). L'instrument d'auto-évaluation a été conçu pour mesurer l'optimisme et la conscience qu'a l'individu de ses capacités d'adaptation à une situation. Il comprend 8 items (quatre positifs et quatre négatifs) sur une échelle de Likert proposant 5 choix de réponse allant de 1 (fortement en accord) à 5 (fortement en désaccord). La cotation de l'instrument est obtenue en attribuant des points à chaque score : $1=4$ points, $2=3$ points, $3=2$ points, $4=1$ point, $5=0$ points. Le total des points détermine le score final de l'optimisme : 32 points indiquaient «le plus optimiste » et 0 《le plus pessimiste ». La fiabilité test-retest (sur un intervalle de 12 mois) a été établie à 0,74 , ce qui tend à prouver que le LOT possède une stabilité raisonnable dans le temps (Carver et al., 1993).

Locus de contrôle. Le locus de contrôle a été mesuré à l'aide du questionnaire $\mathrm{C}$ de l'échelle multidimensionnelle du lieu de contrôle de la santé de Wallston (MHLOC) (Wallston et al., 1994). Le MHLOC est un questionnaire d'auto-évaluation de 18 items, évaluant les croyances de l'individu en ce qui concerne le contrôle qu'il pense pouvoir exercer, notamment sur sa santé. Il comprend deux sous-échelles de 6 items (internalité et externalité aléatoire) et deux souséchelles indépendantes de 3 items (les médecins et d'autres personnes). Chaque item se compose d'une échelle de Likert en six points ( 6 = fortement en accord, $1=$ fortement en désaccord). La somme des scores des items fournit le score total de chaque sous-échelle. Seules les souséchelles d'internalité et d'externalité ont été utilisées dans cette étude.

\section{Procédure}

Après avoir reçu l'approbation du Health Sciences Human Subjects Review Committee de la University at Buffalo - State University of New York, des femmes ont été recrutées par la principale investigatrice dans les six établissements et informées de l'étude, avant d'y accorder leur consentement éclairé. Toutes les données ont été collectées durant le premier cycle de chimiothérapie aux trois intervalles suivants : avant l'administration de la première dose de chimiothérapie, 7 à 14 jours après la première dose (nadir), et à la fin du premier cycle de chimiothérapie. Les informations démographiques (comprenant l'âge, la situation matrimoniale et le statut socio-économique) ont été obtenues dans le dossier médical ou dans le cadre d'entrevues. Les données relatives au niveau de base de l'optimisme, du locus de contrôle et de la détresse liée aux symptômes ont été recueillies dans l'intervalle de temps durant lequel les patientes attendaient leur première dose de chimiothérapie (Temps 1). La détresse liée aux symptômes a été mesurée une nouvelle fois au nadir du premier cycle de chimiothérapie (l'étendue exacte de l'étude était de 9 à 11 jours après le début de la chimiothérapie) (Temps 2), et à la fin du premier cycle de chimiothérapie (approximativement 30 jours après le démarrage du traitement) (Temps 3 ).

\section{Analyse}

L'analyse statistique a été réalisée au moyen du logiciel SPSS Version10.0 pour Windows. Le degré de signification statistique de cette étude a été fixé à 0,05 . Des paramètres descriptifs ont été calculés afin de résumer les variables démographiques ainsi que les

\begin{tabular}{|l|l|l|l|l|}
\hline $\begin{array}{l}\text { Tableau 1 : } \\
\text { Paramètres descriptifs pour les facteurs de personnalité et } \\
\text { les scores de détresse liée aux symptômes T1, T2 T3 }\end{array}$ \\
\hline Variable & $\begin{array}{l}\text { Étendue } \\
\text { possible }\end{array}$ & $\begin{array}{l}\text { Étendue } \\
\text { réelle }\end{array}$ & Moyenne & $\begin{array}{l}\text { Écart- } \\
\text { type }\end{array}$ \\
\hline Optimisme (LOT) & 0 à 32 & 12 à 32 & 23,05 & 4,89 \\
\hline $\begin{array}{l}\text { Locus de contrôle } \\
\text { (Échelle MHLOC- } \\
\text { internalité) }\end{array}$ & 6 à 36 & 6 à 34 & 16,88 & 6,01 \\
\hline $\begin{array}{l}\text { Locus de contrôle } \\
\text { (Échelle MHLOC- } \\
\text { externalité) }\end{array}$ & 6 à 36 & 6 à 36 & 17,76 & 6,03 \\
\hline $\begin{array}{l}\text { Détresse liée aux } \\
\text { symptômes (SDS) T1 }\end{array}$ & 13 à 65 & 17 à 37 & 19,52 & 5,09 \\
\hline $\begin{array}{l}\text { Détresse liée aux } \\
\text { symptômes (SDS) T2 }\end{array}$ & 13 à 65 & 17 à 37 & 24,71 & 4,12 \\
\hline $\begin{array}{l}\text { Détresse liée aux } \\
\text { symptômes (SDS) T3 }\end{array}$ & 13 à 65 & 17 à 37 & 22,91 & 3,32 \\
\hline
\end{tabular}

Tableau 2 : Coefficients de corrélation de Pearson parmi les variables de l'étude

\begin{tabular}{|c|c|c|c|c|c|c|c|c|c|}
\hline & A & SM & $\mathrm{HH}$ & LOT & Int. & Ext. & SDS s & SDS 2 & SDS 3 \\
\hline Âge & 1,00 & & & & & & & & \\
\hline $\begin{array}{l}\text { Situation } \\
\text { matrimoniale }\end{array}$ & $0,32 *$ & 1,00 & & & & & & & \\
\hline $\begin{array}{l}\text { Hollingshead } \\
\text { (SES) }\end{array}$ & $-0,54 *$ & $-0,12$ & 1,00 & & & & & & \\
\hline $\begin{array}{l}\text { Optimisme } \\
\text { ( LOT) }\end{array}$ & $-0,04$ & $-0,06$ & 0,17 & 1,00 & & & & & \\
\hline $\begin{array}{l}\text { Internalité } \\
\text { (MHLOC) }\end{array}$ & $-0,02$ & $-0,03$ & $-0,16$ & 0,09 & 1,00 & & & & \\
\hline $\begin{array}{l}\text { Externalité } \\
\text { (MHLOC) }\end{array}$ & $-0,13$ & $-0,06$ & $-0,08$ & $-0,22 *$ & $-0,06$ & 1,00 & & & \\
\hline Pente du SDS & 0,02 & 0,07 & 0,03 & 0,08 & 0,08 & 0,14 & 1,00 & & \\
\hline SDS total T2 & 0,05 & 0,02 & 0,08 & $-0,27 *$ & $-0,08$ & $-0,16$ & $-0,22 *$ & 1,00 & \\
\hline SDS total T3 & $-0,04$ & 0,03 & 0,16 & $-0,23 *$ & $-0,03$ & $-0,17$ & $-0,02$ & $0,86^{*}$ & 1,00 \\
\hline \multicolumn{10}{|c|}{$\begin{array}{l}\text { a. Pour la situation matrimoniale, on a donné les valeurs nominales } 0=\text { mariée et } \\
1=\text { non mariée } \\
* \mathrm{p}<, 05 \text {. }\end{array}$} \\
\hline
\end{tabular}


scores des variables dépendantes et indépendantes. Les pentes de régression linéaire individuelles de chaque sujet ont été calculées et utilisées comme scores du changement de la détresse liée aux symptômes dans le temps (Kraemer et Thiemann, 1989). Des régressions multiples simultanées ont été calculées en déterminant les régressions des scores du changement du SDS et les scores totaux du SDS à Temps 2 et Temps 3 pour les variables indépendantes de l'âge, de la situation matrimoniale, du niveau socio-économique, de l'optimisme et du locus de contrôle (internalité et externalité).

\section{Résultats}

Caractéristiques de l'échantillon. Lors du recrutement, 137 femmes ont été sollicitées pour participer à l'étude. Neuf d'entre elles ont refusé. Les principales raisons invoquées étaient : «trop de questions », « trop angoissée » ou « j’ai déjà rempli trop de formulaires ». Cinq autres femmes ont commencé à remplir les questionnaires et ont arrêté, ayant jugé les questions trop indiscrètes. Trois sujets qui avaient rempli le questionnaire Temps 1 n'ont pas retourné le questionnaire Temps 2 , et ont par la suite été exclus de l'étude. L'échantillon final se composait de 120 femmes ayant un cancer du sein de stade I ou II, qui suivaient leur premier cycle de chimiothérapie adjuvante. Celles qui avaient refusé de participer à l'étude et/ou s'étaient retirées présentaient les mêmes caractéristiques démographiques et cliniques que ces dernières. Les femmes recrutées pour l'étude étaient en majorité de race blanche (96\%), avaient poursuivi des études au niveau universitaire (80\%) et étaient sur le marché du travail. D'un point de vue clinique, la plupart de ces femmes présentaient un carcinome canalaire infiltrant de stade IA et avaient subi une tumorectomie. Cinquante-deux pour cent n'avaient pas de condition comorbide et avaient toléré la chirurgie sans aucune complication postopératoire. Toutes les patientes avaient été soumises à l'un de ces deux régimes de chimiothérapie : cyclophosphamide, doxorubicine et 5-fluorouracil ou cyclophosphamide, méthotrexate et 5-fluorouracil. La chimiothérapie avait été proposée en option à quarante-deux pour cent d'entre elles, qui avaient choisi de se soumettre au traitement d'une manière préventive. Quarante pour cent des femmes prévoyaient subir une radiothérapie après leur chimiothérapie.

Tableau 3 : Coefficients de régression (bêta) de l'âge, de la situation matrimoniale, du statut socio-économique, de l'optimisme et du locus de contrôle interne et externe vis-àvis de la détresse liée aux symptômes (scores SDS T2, T3 et changement)

\begin{tabular}{|l|l|l|l|}
\hline Variable & $\begin{array}{l}\text { Bêta pour le } \\
\text { score T2 SDS }\end{array}$ & $\begin{array}{l}\text { Bêta pour le } \\
\text { score T3 SDS }\end{array}$ & $\begin{array}{l}\text { Bêta pour } \\
\text { les scores de } \\
\text { changement } \\
\text { SDS }\end{array}$ \\
\hline Âge & 0,12 & 0,02 & 0,08 \\
\hline $\begin{array}{l}\text { Situation } \\
\text { matrimoniale }\end{array}$ & $-0,03$ & 0,02 & 0,07 \\
\hline $\begin{array}{l}\text { Statut socio- } \\
\text { économique (Indice } \\
\text { de Hollingshead) }\end{array}$ & 0,18 & 0,21 & 0,09 \\
\hline Optimisme & $-0,34 *$ & $0,31 *$ & 0,11 \\
\hline $\begin{array}{l}\text { Locus de } \\
\text { contrôle interne }\end{array}$ & $-0,03$ & 0,02 & 0,10 \\
\hline $\begin{array}{l}\text { Locus de } \\
\text { contrôle externe }\end{array}$ & $-0,20 *$ & $-0,22 *$ & $0,19 *$ \\
\hline$*$ p < 0,05. & & & \\
\hline
\end{tabular}

\section{Caractéristiques personnelles et facteurs de personnalité comme prédicteurs de la détresse liée aux symptômes}

Les femmes de l'étude étaient âgées de 37 à 83 ans (moyenne = 59 ; écart-type $=11,62)$ et la plupart étaient mariées $(87 \%)$. Soixantedix-sept pour cent appartenaient à la classe moyenne, selon l'indice d'Hollingshead à deux facteurs, avec un score moyen de 38 (écarttype $=12,4)$ sur une étendue de 8 à 66 . La plupart des femmes de l'étude étaient optimistes, avec un score moyen de 23 (écart-type $=$ 4,9 ) pour une amplitude possible de 1 à 32 . Le locus de contrôle était divisé à parts égales entre internalité $(X=17$; écart-type $=6)$ et externalité $(X=18$; écart-type $=6)$. Les résultats en matière de facteurs de personnalité et de détresse liée aux symptômes (T1, T2, et T3) sont présentés dans le tableau 1.

Afin de déterminer si les facteurs de personnalité et les caractéristiques personnelles sont des prédicteurs de la détresse liée aux symptômes durant le premier cycle de chimiothérapie, des régressions des scores de la détresse liée aux symptômes (total des scores du SDS pour Temps 2, Temps 3 et scores du changement, scores qui reflètent le changement dans les mesures temporelles ) ont été calculées simultanément pour les caractéristiques personnelles, l'optimisme et le locus de contrôle. Les corrélations d'ordre 0 sont montrées dans le tableau 2. Des corrélations inverses significatives sont apparues entre les scores du LOT et du SDS à Temps 2 et Temps 3 ( $r=-0,27$ et $-0,23$ respectivement).

Dans les régressions des scores totaux de la détresse liée aux symptômes Temps 2 pour l'âge, la situation matrimoniale, le statut socio-économique, l'optimisme et le locus de contrôle interne et externe, R2 était 0,15, $(\mathrm{F}=3,21 ; \mathrm{DDL}=6,113 ; \mathrm{p}<0,01)$. L'optimisme et l'externalité ont apporté les seules contributions statistiquement significatives (optimisme : bêta $=-0,34 ; \mathrm{t}=-3,73$; $\mathrm{p}<0,01$; locus de contrôle externe : bêta $=-0,20 ; \mathrm{t}=-2,24 ; \mathrm{p}<0,03$ ) (voir tableau 3).

Dans les régressions des scores totaux de la détresse liée aux symptômes Temps 3 pour l'âge, la situation matrimoniale, le statut socio-économique, l'optimisme et le locus de contrôle interne et externe, R2 était $0,14,(\mathrm{~F}=3,07 ; \mathrm{DDL}=6,113 ; \mathrm{p}<0,01)$. À nouveau, l'optimisme et l'externalité ont apporté les seules contributions significatives (optimisme : bêta $=-0,31 ; \mathrm{t}=-3,41 ; \mathrm{p}<0,01$; externalité : bêta $=-0,21 ; \mathrm{t}=-2.38 ; \mathrm{p}<0,02)$ (tableau 3$)$.

Dans les régressions des scores du changement du SDS pour l'âge, la situation matrimoniale, le statut socio-économique, l'optimisme et le locus de contrôle interne et externe, $\mathrm{R} 2$ était $0,05,(\mathrm{~F}=1,05$; $\mathrm{DDL}=6,113 ; \mathrm{p}<0,05)$. Seul le locus de contrôle externe a donné des résultats statistiquement significatifs (locus de contrôle externe : bêta $=-0,19, \mathrm{t}=1,99, \mathrm{p}<0,05)$ (tableau 3 ).

\section{Les degrés de détresse liée aux symptômes durant le premier cycle de chimiothérapie}

Les scores de la détresse liée aux symptômes étaient généralement bas avec une amplitude potentielle de 13 à 65 et une moyenne de 23 (écart-type $=4,2$ ). Au niveau de référence, les symptômes les plus pénibles étaient l'insomnie, la fatigue et la douleur; au nadir, c'étaient la fatigue, la détresse causée par les changements physiques et l'insomnie. A la fin du premier cycle, on relevait la fatigue, l'insomnie et l'aspect physique. Comme prévu, les scores totaux de la détresse liée aux symptômes étaient plus faibles au début de la chimiothérapie, culminaient au nadir et commençaient à décroître vers la fin du premier cycle du traitement (voir tableau 4). 


\section{Discussion}

Le cadre théorique de Lazarus et Folkman (1984) prédit que la perception du stress et/ou de la détresse chez chaque individu est conditionnelle à son appréciation (évaluation de la situation, stratégies de coping envisagées et prise de conscience) de la situation. Selon cette théorie, on pouvait s'attendre à ce que les caractéristiques personnelles et les facteurs de personnalité prédisent la détresse liée aux symptômes chez les femmes atteintes de cancer du sein. L'hypothèse a été en partie vérifiée dans cette étude, où seuls l'optimisme et le locus de contrôle externe se sont révélés des prédicteurs significatifs d'une faible détresse liée aux symptômes à Temps 2 (nadir) et Temps 3 (fin du cycle), avec des scores élevés pour l'optimisme et le locus de contrôle en corrélation avec des degrés moindres de détresse liée aux symptômes.

La conclusion de cette étude, selon laquelle la détresse liée aux symptômes est plus importante chez les femmes pessimistes, corrobore les travaux de Carver (1993), qui ont démontré que le pessimisme augmentait le risque de réactions psychologiques négatives au diagnostic et au traitement de la maladie chez des femmes atteintes de cancer du sein. La découverte significative de la relation entre le locus de contrôle externe et le faible degré de détresse liée aux symptômes ne confirme pas les résultats décrits par Ehlke (1988), associant le locus de contrôle interne aux faibles scores de détresse liée aux symptômes. Les femmes dans son étude avaient le sentiment d'exercer un certain contrôle sur la situation, alors que les femmes de la présente étude avaient une attitude plus fataliste et affichaient une plus grande confiance dans les prestataires de soins.

Aucune des caractéristiques personnelles étudiées ne prédisait la détresse liée aux symptômes, contrairement à des recherches antérieures, où les patientes plus jeunes présentaient des degrés plus élevés de détresse liée aux symptômes (Cimprich, 1999; Degner et Sloan, 1995; Pasacreta, 1997; Sarna et Brecht, 1997) et où le soutien du conjoint/de la famille contribuait à réduire les niveaux de détresse liée aux symptômes (Northouse, Dorris et Charron-Moore, 1995; Tishelman, Taube et Sachs, 1991). Cette dernière contradiction peut s'expliquer par le fait que $87 \%$ des femmes de cette étude étaient mariées et avouaient pouvoir se confier à quelqu'un.

Les modestes scores sur l'indice d'Hollingshead indiquent que la plupart des femmes de cette étude avaient un bon niveau d'éducation et appartenaient à la classe moyenne. Aucune relation n'a pu être établie entre le statut socio-économique et les degrés de détresse liée aux symptômes observés. Signalons, à titre d'information, que les qualités psychométriques de validité et/ou de fiabilité ont été publiées à l'aide de cet instrument.

L'absence de relation entre les caractéristiques personnelles et la détresse liée aux symptômes peut être attribuée au fait que la population cible de cette étude ne comprenait que des femmes atteintes d'un cancer du sein, ce qui limitait la variabilité du genre et du diagnostic. Dans plusieurs études antérieures, le genre et le diagnostic n'étaient pas contrôlés. La variabilité généralement faible des scores pourrait également expliquer l'absence de lien significatif entre la détresse liée aux symptômes et le statut socioéconomique. La taille de l'échantillon constitue une autre explication possible quant à la disparité des résultats concernant les caractéristiques personnelles et la détresse liée aux symptômes entre cette étude et les recherches antérieures. En effet, les études précédentes faisant état de corrélations entre diverses caractéristiques personnelles et la détresse liée aux symptômes portaient sur des échantillons plus restreints (Tishelman, 1991; DeKeyser, 1998).

Les caractéristiques personnelles, l'optimisme et le locus de contrôle n'ont pas permis de prédire le changement de la détresse liée aux symptômes entre les trois moments où les mesures ont été effectuées durant le premier cycle de chimiothérapie. On peut attribuer cela aux degrés de changement généralement faibles de la détresse liée aux symptômes rapportée par les sujets sur toute la durée de la période concernée.

Cette étude a aussi examiné la détresse liée aux symptômes ressentie durant le premier cycle de chimiothérapie. Bien que la plupart des femmes de cette étude aient été considérées relativement en bonne santé, toutes ont éprouvé un certain degré de détresse liée aux symptômes. Comme prévu, les degrés de détresse les plus élevés ont été atteints au nadir du cycle de chimiothérapie, néanmoins les scores de cette étude étaient généralement bas et corroboraient les conclusions d'Ehlke (1988) et de Boehmke (2004). Ces faibles scores peuvent être attribués aux sentiments et aux expériences de ces femmes, qui avaient suivi des protocoles de chimiothérapie relativement faciles à supporter, ou au manque de précision de la mesure de la détresse liée aux symptômes au moyen des instruments actuels (Ehlke, 1988; Boehmke, 2004).

\section{Limites de l'étude}

Malgré la sélection de six établissements socialement diversifiés, l'échantillon de convenance traduisait une population homogène. Les femmes étaient essentiellement blanches, mariées et issues de la classe moyenne. Cette homogénéité de l'échantillon limite la généralisation de l'étude. L'usage de médicaments, y compris des anti-émétiques et des antidépresseurs, n'a pas été mesuré ni contrôlé dans cette étude et n'est peut-être pas étranger aux scores globalement faibles de la détresse liée aux symptômes. Les sujets n'ont été suivis que durant le premier cycle de chimiothérapie adjuvante, et bien que

\section{Tableau 4 : Moyennes lors des Temps 1, 2 et 3 pour chaque élément relatif à la détresse liée aux symptômes, obtenues} grâce à l'échelle de McCorkle

\begin{tabular}{|l|l|l|l|}
\hline & $\begin{array}{l}\text { T1 } \\
\text { Moyenne } \\
\text { (SD) }\end{array}$ & $\begin{array}{l}\text { T2 } \\
\text { Moyenne } \\
\text { SD })\end{array}$ & $\begin{array}{l}\text { T3 } \\
\text { Moyenne } \\
\text { (SD) }\end{array}$ \\
\hline $\begin{array}{l}\text { Fatigue } \\
(0,568)\end{array}$ & $1,98(0,730)$ & $2,95(0,482)$ & 20,38 \\
\hline Apparence & $1,43(0,666)$ & $2,55(0,563)$ & $2,13(0,387)$ \\
\hline Insomnie & $2,02(1,016)$ & $2,47(0,819)$ & $2,25(0,770)$ \\
\hline $\begin{array}{l}\text { Confiance } \\
\text { en l'avenir }\end{array}$ & $1,75(0,635)$ & $2,40(0,556)$ & $2,03(0,533)$ \\
\hline Concentration & $1,48(0,739)$ & $2,32(0,663)$ & $1,97(0,614)$ \\
\hline $\begin{array}{l}\text { Apparition de } \\
\text { la nausée }\end{array}$ & $1,28(0,566)$ & $2,27(0,576)$ & $2,07(0,370)$ \\
\hline $\begin{array}{l}\text { Gravité de } \\
\text { la nausée }\end{array}$ & $1,36(0,703)$ & $2,05(0,548)$ & $1,70(0,630)$ \\
\hline Perte d'appétit & $1,33(0,582)$ & $2,04(0,353)$ & $1,85(0,461)$ \\
\hline $\begin{array}{l}\text { Apparition de } \\
\text { douleurs }\end{array}$ & $1,80(1,005)$ & $1,66(0,845)$ & $1,56(0,838)$ \\
\hline $\begin{array}{l}\text { Gravité des } \\
\text { douleurs }\end{array}$ & $1,35(0,558)$ & $1,42(0,602)$ & $1,32(0,518)$ \\
\hline Troubles du transit & $1,32(0,631)$ & $1,27(0,576)$ & $1,26(0,572)$ \\
\hline Toux & $1,28(0,548)$ & $1,23(0,514)$ & $1,22(0,476)$ \\
\hline Respiration & $1,15(0,474)$ & $1,15(0,461)$ & $1,17(0,455)$ \\
\hline $\begin{array}{l}\text { Note : Pour chaque élément, l'étendue éventuelle variait de } 1= \\
\text { détresse liée aux symétrese liée aux symptômes à 5 = Plus fort degré de }\end{array}$ \\
\hline
\end{tabular}


la force de cette approche réside dans le fait que peu d'entre eux en ont été exclus pour cause de neutropénie, d'infection ou de délais de traitement, le choix de cette période relativement courte ne fournit qu'un bref aperçu de l'expérience des femmes suivant une chimiothérapie adjuvante pour un cancer du sein.

\section{Implications pour la recherche future}

La reproduction de cette étude avec un échantillon plus diversifié sur une période plus longue fournirait davantage d'informations utiles sur la détresse liée aux symptômes qu'éprouvent actuellement les femmes atteintes de cancer du sein. Des enquêtes approfondies sur le rôle de l'optimisme et du locus de contrôle s'imposent. Une étude qualitative apporterait des précisions sur les symptômes tels qu'ils sont ressentis et sur la sévérité de la détresse liée aux symptômes véritablement éprouvée par les femmes atteintes de cancer du sein, sans qu'elle soit reliée aux questions particulières d'un instrument quantitatif. Le traitement adjuvant du cancer du sein est appelé à évoluer, au fur et à mesure que de nouveaux agents chimiothérapiques viendront s'ajouter aux protocoles de traitement, provoquant des symptômes et une détresse liée aux symptômes différents des précédents. Des plaintes de douleurs aux os et aux articulations, de neuropathies diverses et de symptômes ménopausiques sont indissociables des protocoles actuels de chimiothérapie; aucun de ces symptômes n'est pourtant mesuré par les instruments d'évaluation de la détresse liée aux symptômes disponibles, dont un grand nombre ont été créés dans les années 70 et 80 . La disparité des symptômes ressentis et des symptômes mesurés justifie l'élaboration d'un outil plus précis pour mesurer la détresse liée aux symptômes chez les femmes d'aujourd'hui atteintes de cancer du sein.

\section{References}

Boehmke, M.M. (2004). Measurement of symptom distress in women with early-stage breast cancer. Cancer Nursing, 27(2), 144-152.

Carver, C.S., Pozo-Kaderman, C., Harris, S.D., Noriega, V., Scheier, M.F., Robinson, D.S., et al. (1993). Optimism versus pessimism predicts the quality of women's adjustment to early stage breast cancer. Cancer, 73(4), 1213-1220.

Cimprich, B. (1999). Pretreatment symptom distress in women newly diagnosed with breast cancer. Cancer Nursing, 22(3), 185-194.

Cohen, J. (1992). A power primer. Psychological Bulletin, 112(1), 155-159.

Degner, L.F., \& Sloan, J.A. (1995). Symptom distress in newly diagnosed ambulatory cancer patients and as a predictor of survival in lung cancer. Journal of Pain and Symptom Management, 10(6), 423-431.

DeKeyser, F.G., Wainstock, J.M., Rose, L., Converse, P.J., \& William, D. (1998). Distress, symptom distress, and immune function in women with suspected breast cancer. Oncology Nursing Forum, 25(8), 1415-1421.

Ehlke, G. (1988). Symptom distress in breast cancer patients receiving chemotherapy in the outpatient setting. Oncology Nursing Forum, 15(3), 343-346.

Hollingshead, A.B. (1975). Four-factor index of social status. Unpublished manuscript, Yale University, Department of Sociology.

Kraemer, H.C., \& Thiemann, S. (1989). A strategy to use soft data effectively in randomized controlled clinical trials. Journal of Consulting and Clinical Psychology, 57, 148-154.

Kukull, W., McCorkle, R., \& Driever, M. (1986). Symptom distress, psychosocial variables, and survival from lung cancer. Journal of Psychosocial Oncology, 4(1/2, Spring/Summer), 91-104.

Lazarus, R.S. (1966). Psychological stress and the coping process. New York: McGraw-Hill Book Company.

\section{Implications cliniques}

Développer un sens de l'optimisme et du contrôle - deux variables présentées dans cette étude comme fortement en lien avec les niveaux de détresse liée aux symptômes éprouvée contribuerait à améliorer la qualité de vie des femmes à risque ainsi que leur expérience du cancer. La fatigue, le changement de l'apparence physique et l'insomnie étaient les symptômes associés aux seuils de détresse les plus élevés durant le premier cycle de chimiothérapie. Bien que dans cette étude, les femmes aient rapporté des degrés modestes de détresse liée aux symptômes, les prestataires de soins ne devraient pas les ignorer, car même les femmes qui étaient considérées en bonne santé avant que leur cancer du sein ne soit diagnostiqué ont éprouvé des niveaux plus élevés de détresse liée aux symptômes lorsqu'elles ont commencé à subir les effets de la chimiothérapie autour du nadir (approximativement le 10e jour du cycle). La connaissance des moments où la détresse liée aux symptômes atteint son paroxysme durant le premier cycle de chimiothérapie fournit aux prestataires de soins le cadre temporel où il est important d'informer et d'éduquer les patientes, avant le pic des symptômes, et promouvoir l'empowerment pour qu'elles deviennent de véritables partenaires de leur équipe soignante. Dépister celles qui présentent le plus grand risque de développer des niveaux élevés de détresse liée aux symptômes peut aider les prestataires de soins à leur indiquer les manières de gérer les symptômes pénibles, avant que ceux-ci et la détresse qui y est associée ne surviennent.

Lazarus, R.S., \& Folkman, S. (1984). Stress, appraisal, and coping New York: Springer Publishing Company.

McCorkle, R., \& Young, K. (1978, October). Development of symptom distress scale. Cancer Nursing, 373-378.

Munkres, A., Oberst, M.T., \& Hughes, S.H. (1992). Appraisal of illness, symptom distress, self-care burden, and mood states in patients receiving chemotherapy for initial and recurrent cancer. Oncology Nursing Forum, 19(8), 1201-1209.

Northouse, L.L., Dorris, G., \& Charron-Moore, C. (1995). Factors affecting couples' adjustment to recurrent breast cancer. Social Science Medicine, 41(1), 69-76.

Pasacreta, J.V. (1997). Depressive phenomena, physical symptom distress, and functional status among women with breast cancer. Nursing Research, 46(4), 214-221.

Rhodes, V.A., \& McDaniel, R.W. (1999). The symptom experience and its impact on quality of life. In C. Henke Yarbro, M. Hanson Frogge, \& M. Goodman (Eds.), Cancer Symptom Management (pp. 3-9). Boston: Jones and Bartlett Publishers.

Rhodes, V.A., \& Watson, P.M. (1987). Symptom distress - the concept: Past and present. Seminars in Oncology Nursing, 3(4), 242-247.

Sarna, L., \& Brecht, M.L. (1997). Dimensions of symptom distress in women with advanced lung cancer: A factor analysis. Heart \& Lung, 26(1), 23-30.

Scheier, M.F., \& Carver, C.S. (1985). Optimism, coping, and health: Assessment and implications of generalized outcome expectancies. Health Psychology, 4(3), 219-247.

SPSS Inc. (2001, July 25). SPSS for Windows Release 10.00 [Computer software]. Chicago, IL: SPSS Inc.

Tishelman, C., Taube, A., \& Sachs, L. (1991). Self-reported symptom distress in cancer patients: Reflections of disease, illness or sickness? Social Science Medicine, 33(11), 1229-1240. 